\title{
Predictive Value of Sudden Olfactory Loss in the Diagnosis of COVID-19
}

\author{
Antje Haehner ${ }^{a} \quad$ Julia Draf ${ }^{a}$ Sarah Dräger ${ }^{b}$ Katja de With ${ }^{b}$ \\ Thomas Hummel ${ }^{\mathrm{a}}$ \\ a Smell and Taste Clinic, Department of Otorhinolaryngology, TU Dresden, Dresden, \\ Germany; ${ }^{b}$ Division of Infectious Diseases, TU Dresden, Dresden, Germany
}

\section{Keywords}

COVID-19 $\cdot$ SARS-CoV-2 $\cdot$ Smell $\cdot$ Olfactory loss $\cdot$ Diagnostic accuracy

\begin{abstract}
Introduction: Recent reports suggest that sudden smell loss might be a symptom of SARS$\mathrm{CoV}-2$ infection. The aim of this study was to investigate the frequency of olfactory loss in an outpatient population who presented to a coronavirus testing center during a 2-week period and to evaluate the diagnostic value of the symptom "sudden smell loss" for screening procedures. Methods: In this cross-sectional controlled cohort study, 500 patients who presented with symptoms of a common cold to a corona testing center and fulfilled corona testing criteria completed a standardized diagnostic questionnaire which included the patients' main symptoms, time course, and an additional self-assessment of the patients' current smell, taste function, and nasal breathing compared to the level before the onset of symptoms. Results: Out of the 500 patients, 69 presented with olfactory loss. Twenty-two of them subsequently tested positive for SARS-CoV-2. Only 12 out of the patients without olfactory loss tested positive, resulting in a frequency of $64.7 \%$ for the symptom "sudden smell loss" in COVID-19 patients. Compared to COVID-19 patients without smell loss, they were significantly younger and less severely affected. Changes in nasal airflow were significantly more pronounced in SARSCoV-2 negative patients with olfactory complaints compared to the patients with smell loss who tested positive for SARS-CoV-2. By excluding patients with a blocked nose, the symptom "sudden smell loss" can be attested a high specificity (97\%) and a sensitivity of $65 \%$ with a positive predictive value of $63 \%$ and negative predictive value of $97 \%$ for COVID-19. Conclusion: Considering the high frequency of smell loss in non-hospitalized COVID-19 patients, acute olfactory impairment should be recognized as an early symptom of the disease and should be tested for on a regular basis. In contrast to other acute viral smell impairment, COVID-19-associated smell loss seems to be only rarely accompanied by a severely blocked nose.
\end{abstract}


Haehner et al.: Olfactory Loss in the Diagnosis of COVID-19

\section{Introduction}

Viral upper respiratory tract infections (URTI) are one of the most common causes of olfactory loss. Together with rhino-, adeno-, influenza-, and parainfluenza viruses, coronaviruses have long been known to account for at least $70 \%$ of common colds [1]. Compared to other respiratory viruses however, the frequency of reported olfactory disorders in coronavirus infections has been lower so far and has never been a serious health issue [2-4].

During an acute URTI, nasal congestion and rhinorrhea are commonly accompanied by a temporary smell loss of varying severity. Significant olfactory loss without rhinitic symptoms, however, is comparatively rare and points towards damage of the olfactory epithelium and/ or the olfactory bulb. URTI-associated smell loss typically occurs after the fifth decade of life and becomes apparent to the patients several days after the onset of a cold [5]. According to a number of anecdotal reports and a few published studies, a different pattern is seen in patients with severe acute respiratory syndrome coronavirus 2 (SARS-CoV-2) infection. Smell and/or taste loss have been observed in $5-80 \%$ of these patients [6-9], sometimes as the only apparent symptom, at a younger age, and at a very early stage of the disease. According to the latest research findings, SARS-CoV-2 is thought to target non-neuronal support cells, as well as the neuroregenerative basal cell population, due to their expression of the receptor ACE2 [10]. Disruption of these cell populations may impair olfactory receptor neuron function and provoke subsequent olfactory receptor neuron damage which might cause olfactory dysfunction. The diagnostic accuracy of this symptom, however, is controversially discussed considering the fact that more than 200 viruses are known to cause URTIs with a subsequent olfactory loss.

The aim of this study was to investigate the frequency of olfactory loss in an outpatient population who presented to a coronavirus testing center during a 2-week period and to evaluate the diagnostic value of this symptom for screening procedures.

\section{Materials and Methods}

All patients who presented to the coronavirus testing center at the University Hospital Dresden routinely received a standardized diagnostic questionnaire which included the patients' main symptoms, time course, and an additional self-assessment of the patients' current smell, taste function, and nasal breathing compared to the level before the onset of symptoms. The patients had to indicate whether they experienced loss of smell and/or taste (yes vs. no). For quantifying olfactory/gustatory function and nasal breathing, a visual analog scale (VAS), with the extreme left of the scale defined as "no function" (0 units) and the extreme right of the scale defined as highest function possible ("extremely good" - 10 units), was used.

Out of 620 patients who fully completed the diagnostic questionnaire, 500 met the criteria for SARSCoV-2 testing, and throat swabs were collected according to WHO recommendations [11]. In 34 patients (6.8\%), the diagnosis of SARS-CoV-2 infection was confirmed by means of a positive RT-PCR test.

\section{Results}

\section{Study Population}

Out of the 500 patients (mean age, 41.3 years; range, 18-86 years) who presented with symptoms of a common cold (mean duration, 5.8 days; range, 1-32 days) and fulfilled corona testing criteria, $45.4 \%$ were male and $54.6 \%$ female. The main symptoms were cough $(76.8 \%)$, sore throat (64.8\%), rhinorrhea (55.2\%), myalgia (40.0\%), dyspnea (33.4\%), and fever (20.8\%). Sixty-nine patients (13.8\%) reported sudden smell and/or taste loss. 
Haehner et al.: Olfactory Loss in the Diagnosis of COVID-19

Table 1. Patient characteristics

\begin{tabular}{llll}
\hline & $\begin{array}{l}\text { Correct } \\
(n=22)\end{array}$ & $\begin{array}{l}\text { False negative } \\
(n=12)\end{array}$ & $\begin{array}{l}\text { False positive } \\
(n=47)\end{array}$ \\
\hline Female gender & 54.5 & 33.3 & 65.2 \\
Age, years & $38.6 \pm 12.4$ & $47.9 \pm 10.8$ & $39.3 \pm 12.4$ \\
Symptoms onset before testing, days & $5.5 \pm 3.2$ & $4.3 \pm 2.9$ & $7.0 \pm 4.9$ \\
Smell/taste loss onset before testing, days & $3.1 \pm 3.3$ & & $5.4 \pm 3.8$ \\
Smell VAS score difference & $-6.8 \pm 2.3$ & 0 & $-5.3 \pm 1.9$ \\
Nasal breathing VAS score difference & $-1.1 \pm 1.6$ & $-0.6 \pm 0.9$ & $-4.9 \pm 2.4$ \\
Cough & 81.0 & 75.0 & 91.5 \\
Sore throat & 42.9 & 58.3 & 83.0 \\
Dyspnea & 19.0 & 25.0 & 59.6 \\
Fever & 19.0 & 58.3 & 23.4 \\
Rhinorrhea & 45.5 & 33.3 & 80.9 \\
Myalgia & 38.1 & 50.0 & 55.3 \\
\hline
\end{tabular}

Data are presented as $\%$ or mean \pm SD, as appropriate. COVID-19 patients with (correct) and without olfactory loss (false negative) and SARS-CoV-2-negative patients with smell loss (false positive) out of a total patient population of $n=500$.

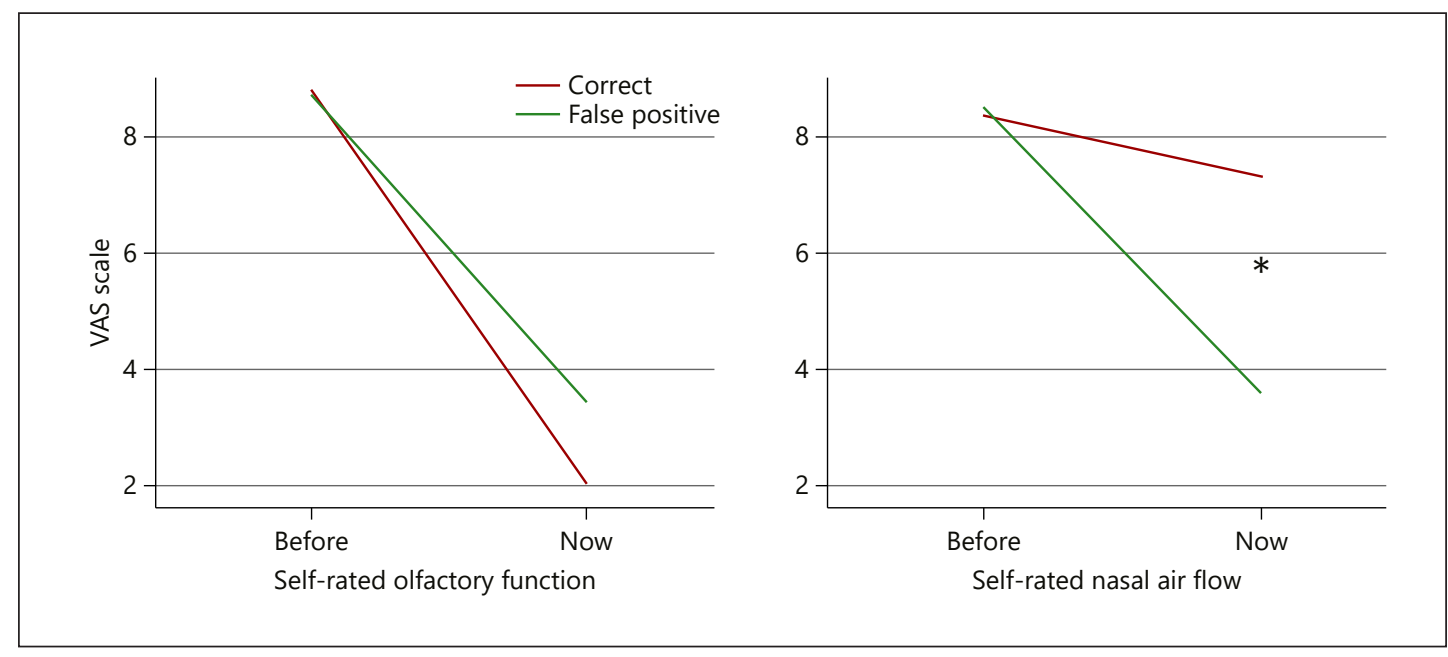

Fig. 1. Change in self-rated olfactory function and nasal breathing compared to the level before disease in SARS-CoV-2-positive (correct) and SARS-CoV-2-negative patients with smell loss (false positive).

\section{COVID-19 Patients}

Out of the 34 patients who tested positive for SARS-CoV-2, 22 (64.7\%) complained of sudden smell and taste loss. The chemosensory loss started 1-2 days before other symptoms in 1 patient, at the same time in 4 patients, and 1-7 days after in 14 patients; 3 patients were not sure about the smell loss onset. COVID-19 patients with olfactory loss were significantly younger than those without smell loss $(\mathrm{p}=0.04)$ and had less severe symptoms (Table 1$)$. Smell loss in COVID-19 patients was characterized by a severe loss of function from an average of 8.8 to 2 and minor changes in nasal breathing from an average of 8.4 to 7.3, as rated on the 10-point VAS. Only 10 patients complained of rhinorrhea.

While only one patient complained about isolated taste loss, the majority of patients reported either combined smell and taste loss, or smell loss only. 
Haehner et al.: Olfactory Loss in the Diagnosis of COVID-19

\section{Patients with Smell Loss without SARS-CoV-2 Infection}

Four hundred sixty-six patients tested negative for SARS-CoV-2, 47 of whom (10.1\%) reported disorder-associated smell loss. Olfactory impairment started 1-14 days after the other symptoms had begun and was characterized by both severe loss of function (mean value, from 8.8 to 3.4) and impaired nasal breathing (mean value, from 8.5 to 3.6) as rated on the 10-point VAS. The majority of these patients (80.9\%) suffered from rhinorrhea (Table 1).

Disease-associated changes in nasal airflow were significantly more pronounced in the 47 SARS-CoV-2-negative patients with olfactory complaints compared to the 22 patients with smell loss who tested positive for SARS-CoV-2 ( $p<0.001)$ (Fig. 1). In contrast, changes in olfactory function were rated more severe in the latter group $(p=0.023)$.

\section{Predictive Value}

Using our data of an outpatient population, the sole symptom "smell loss" had the following diagnostic characteristics: $65 \%$ sensitivity, $90 \%$ specificity, $32 \%$ positive predictive value (PPV), and $97 \%$ negative predictive value (NPV). Taking into account the low frequency of sinunasal symptoms in COVID-19 patients and excluding those with a blocked nose from the false-positive sample, however, PPV increased to $63 \%$ and specificity to $97 \%$.

\section{Discussion}

In this large URTI patient cohort study of a coronavirus testing center, we found that $13 \%$ of patients reported sudden olfactory loss. Of these patients, $32 \%$ subsequently tested positive for SARS-CoV-2. On the other hand, $2.8 \%$ of the patients without olfactory loss tested positive.

In this outpatient sample, $64.7 \%$ of COVID-19 patients observed smell loss at a very early stage or even before other symptoms started. The frequency is much higher compared to the observations by Giacomelli et al. [8], but slightly below the results reported by Lechien et al. [7] and Vaira et al. [9], taking into account that varying specimen selection (nasopharyngeal vs. throat swabs) with different viral loads was applied in these studies [12]. Compared to COVID-19 patients without smell loss, in our study COVID-19 patients with smell dysfunction were significantly younger and less severely affected. Similar results were reported in an Italian study where chemosensory impairment was more frequently seen amongst younger COVID-19 patients [8]. In our study, more than $80 \%$ of COVID-19 patients with smell loss also reported taste dysfunction while isolated taste loss was not observed. The latter impairment might, however, be attributed to impaired retronasal olfaction (flavor) rather than impaired gustation (sweet, salty, sour, bitter, and umami). This is in line with recent assessment-based results [9] and points towards a primary olfactory deficit.

In contrast to other acute viral smell impairment, COVID-19-associated smell loss in our study was only rarely accompanied by a severely blocked nose, and thus the effect on olfaction may be independent of nasal congestion. This complies with the results by Vaira et al. [9] who observed nasal obstruction and rhinitic symptoms in only $9.1 \%$ of their COVID-19 cases. By taking into account these findings and excluding patients with a markedly blocked nose from our data set, the symptom "sudden smell loss" can be attested a high specificity (97\%) and sensitivity of $65 \%$ with a PPV of $63 \%$ and NPV of $97 \%$ for COVID-19. Therefore, olfactory function should be measured in all patients with suspected COVID-19 for which fast, inexpensive, and reliable screening methods are available (e.g., disposable Q-Sticks tests [13]; for a possible approach see also [14]). Further, sudden smell loss without nasal congestion might be used as an indicator for home quarantine in countries were tests are not available in view of the comparable sensitivity and specificity of swab tests [15]. 
Haehner et al.: Olfactory Loss in the Diagnosis of COVID-19

\section{Conclusion}

Thus, considering the high frequency of sudden smell loss in non-hospitalized COVID-19 patients, acute olfactory impairment should be recognized as an early symptom of the disease. It also suggests the need for an exhaustive olfactory and gustatory follow-up of these patients in order to assess the extent of the olfactory loss and the significance of the chemosensory loss in terms of the prognosis of the patients.

\section{Statement of Ethics}

Investigations were performed according to the Guidelines for Biomedical Studies Involving Human Subjects (Helsinki Declaration). The protocol was approved by the Ethics Committee of the Medical Faculty of the TU Dresden (EK-115032020), and all subjects provided written informed consent.

\section{Disclosure Statement}

The authors declare no potential conflicts of interest with respect to the research, authorship, and/or publication of this article.

\section{Funding Sources}

There is no sponsorship or funding arrangement related to this research.

\section{Author Contributions}

Antje Haehner: study conception; data acquisition, analysis, and interpretation; drafting, revision, and final approval of the manuscript. Julia Draf: data acquisition and interpretation; revision and final approval of the manuscript. Sarah Dräger and Katja de With: data acquisition and interpretation; revision and final approval of the manuscript. Thomas Hummel: study conception; data analysis and interpretation; drafting, revision, and final approval of the manuscript.

\section{References}

1 Heikkinen T, Järvinen A. The common cold. Lancet. 2003 Jan;361(9351):51-9.

2 Sugiura M, Aiba T, Mori J, Nakai Y. An epidemiological study of postviral olfactory disorder. Acta Otolaryngol Suppl. 1998;538:191-6.

3 Konstantinidis I, Haehner A, Frasnelli J, Reden J, Quante G, Damm M, et al. Post-infectious olfactory dysfunction exhibits a seasonal pattern. Rhinology. 2006 Jun;44(2):135-9.

4 Suzuki M, Saito K, Min WP, Vladau C, Toida K, Itoh H, et al. Identification of viruses in patients with postviral olfactory dysfunction. Laryngoscope. 2007 Feb;117(2):272-7.

5 Hummel T, Whitcroft KL, Andrews P, Altundag A, Cinghi C, Costanzo RM, et al. Position paper on olfactory dysfunction. Rhinol Suppl. 2017 Mar;54(26):1-30.

6 Mao L, Wang M, Chen S, Hu Y, Chen S, He Q, et al. Neurological manifestations of hospitalized patients with coronavirus disease 2019 in Wuhan, China. JAMA Neurol. 2020 Apr. Epub ahead of print. Available from. https://doi.org/10.1001/jamaneurol.2020.1127.

7 Lechien JR, Chiesa-Estomba CM, De Siati DR, Horoi M, Le Bon SD, Rodriguez A, et al. Olfactory and gustatory dysfunctions as a clinical presentation of mild-to-moderate forms of the coronavirus disease (COVID-19): a multicenter European study. Eur Arch Otorhinolaryngol. 2020 Apr. Epub ahead of print. Available from: https://doi.org/10.1007/s00405-020-05965-1.

8 Giacomelli A, Pezzati L, Conti F, Bernacchia D, Siano M, Oreni L, et al. Self-reported olfactory and taste disorders in SARS-CoV-2 patients: a cross-sectional study. Clin Infect Dis. 2020 Mar:ciaa330. Epub ahead of print.

9 Vaira LA, Deiana G, Fois AG, Pirina P, Madeddu G, De Vito A, et al. Objective evaluation of anosmia and ageusia in COVID-19 patients: single-center experience on 72 cases. Head Neck. 2020 Jun;42(6):1252-8. ; Epub ahead of print. 
Haehner et al.: Olfactory Loss in the Diagnosis of COVID-19

10 Brann D, Tsukahara T, Weinreb C, Logan D, Datta S. Non-neural expression of SARS-CoV-2 entry genes in the olfactory epithelium suggests mechanisms underlying anosmia in COVID-19 patients. bioRxiv. 2020. Available from: https://doi.org/10.1101/2020.03.25.009084.

11 WHO. COVID-19 - technical guidance [accessed 2020 Apr 6]. Available from: https://www.who.int/emergencies/diseases/novel-coronavirus-2019/technical-guidance.

12 Zou L, Ruan F, Huang M, Liang L, Huang H, Hong Z, et al. SARS-CoV-2 viral load in upper respiratory specimens of infected patients. N Engl J Med. 2020 Mar;382(12):1177-9.

13 Sorokowska A, Oleszkiewicz A, Minovi A, Konnerth CG, Hummel T. Fast Screening of Olfactory Function Using the Q-Sticks Test. ORL J Otorhinolaryngol Relat Spec. 2019;81(5-6):245-51.

14 Whitcroft KL, Hummel T. Olfactory Dysfunction in COVID-19: diagnosis and Management. Epub ahead of print. JAMA. 2020 May. Available from: https://doi.org/10.1001/jama.2020.8391.

15 Watson J, Whiting PF, Brush JE. Interpreting a COVID-19 test result. BMJ. 2020 May;369:m1808. 